\title{
As alterações oftalmológicas pelo uso de cloroquina e/ou hidroxicloroquina no contexto da COVID-19
}

The ophthalmological alterations due to the use of chloroquine and/or hydroxychlroquine in the context of the COVID-19

Los cambios oftalmológicos debidos al uso de cloroquina y/o hidroxicloroquina en el contexto de la COVID-19

\section{Resumo}

No contexto da pandemia do novo coronavírus, as drogas cloroquina e hidroxicloroquina foram divulgadas massivamente e utilizadas como tratamento contra a COVID-19. Sabe-se que esses medicamentos possuem efeitos colaterais, dentre eles complicações oculares. O presente estudo analisa se é possível a ocorrência desses efeitos colaterais oftalmológicos, considerando a prescrição destes fármacos recomendada para o tratamento precoce da COVID-19 pelo Ministério da Saúde do Brasil em 2020. Este trabalho tem por objetivo realizar uma revisão integrativa no intuito de identificar quais são os efeitos colaterais oftalmológicos pelo uso de cloroquina (CQ) e hidroxicloroquina (HCQ). Com busca na plataforma PUBMED. O processo utilizou como termos de busca "Hidroxicloroquina", "Cloroquina", "Oftalmopatia" e "Retinopatia". Incluídos artigos científicos publicados entre 2015 e 2020, em inglês ou português e de acesso livre ao texto integral. Foram coletados dados referentes aos autores, principais resultados, fatores de risco e ano da publicação. Foram selecionados 14 estudos, em que toxicidade retinal ou retinopatia por HCQ/CQ são abordadas. Foi observado que os fatores de risco mais importantes o desenvolvimento das complicações oculares são uma dose acumulativa de, ao menos, 600g de HCQ ou de, pelo menos, 460g de CQ e uma terapia com duração de, no mínimo, 3 anos. Além disso, foram expostos outros fatores de risco como a dose diária ingerida, tipo físico, uso concomitante de tamoxifeno e as comorbidades dos pacientes. O desenvolvimento da retinopatia por hidroxicloroquina e cloroquina é dependente, principalmente, da dose acumulativa e da duração da terapia.

Palavras-chave: Hidroxicloroquina; Cloroquina; Retinopatia; COVID-19.

\footnotetext{
Abstract

In the context of the new coronavirus pandemic, the drugs chloroquine and hydroxychloroquine were massively disseminated and used as a treatment against COVID-19. These drugs are known to have side effects, including ocular complications. The present study analyzes whether these ophthalmic side effects are possible, considering the prescription of these drugs recommended for the early treatment of COVID-19 by the Ministry of Health of Brazil in 2020. This work aims to carry out an integrative review in order to identify what are the ophthalmologic side effects from the use of chloroquine (CQ) and hydroxychloroquine (HCQ). Searching on the PUBMED platform. The process used as search terms "Hydroxychloroquine", "Chloroquine", "Ophthalmopathy" and "Retinopathy". Included
} 
scientific articles published between 2015 and 2020, in English or Portuguese and with free access to the full text. Data regarding authors, main results, risk factors and year of publication were collected. Fourteen studies were selected, in which retinal toxicity or HCQ/CQ retinopathy are addressed. It was observed that the most important risk factors for the development of ocular complications are a cumulative dose of at least 600g of HCQ or of at least $460 \mathrm{~g}$ of CQ and a therapy lasting at least 3 years. In addition, other risk factors were exposed, such as daily intake, physical type, concomitant use of tamoxifen and patients' comorbidities. The development of hydroxychloroquine and chloroquine retinopathy is mainly dependent on the cumulative dose and duration of therapy.

Keywords: Hydroxychloroquine; Chloroquine; Retinopathy; COVID-19.

\section{Resumen}

En el contexto de la nueva pandemia de coronavirus, los fármacos cloroquina e hidroxicloroquina se difundieron masivamente y se utilizaron como tratamiento contra COVID-19. Se sabe que estos medicamentos tienen efectos secundarios, incluidas complicaciones oculares. El presente estudio analiza si estos efectos secundarios oftálmicos son posibles, considerando la prescripción de estos medicamentos recomendados para el tratamiento temprano de COVID-19 por el Ministerio de Salud de Brasil en 2020. Este trabajo tiene como objetivo realizar una revisión integradora con el fin de identificar ¿Cuáles son los efectos secundarios oftalmológicos del uso de cloroquina (CQ) e hidroxicloroquina (HCQ)? Buscando en la plataforma PUBMED. El proceso utilizó como términos de búsqueda "hidroxicloroquina", "cloroquina”, “oftalmopatía” y "retinopatía". Se incluyeron artículos científicos publicados entre 2015 y 2020, en inglés o portugués y con acceso gratuito al texto completo. Se recogieron datos sobre autores, principales resultados, factores de riesgo y año de publicación. Se seleccionaron catorce estudios, en los que se abordan la toxicidad retiniana o la retinopatía por HCQ / CQ. Se observó que los factores de riesgo más importantes para el desarrollo de complicaciones oculares son una dosis acumulada de al menos $600 \mathrm{~g}$ de HCQ o de al menos 460 g de CQ y una terapia que dure al menos 3 años. Además, se expusieron otros factores de riesgo, como la ingesta diaria, el tipo físico, el uso concomitante de tamoxifeno y las comorbilidades de los pacientes. El desarrollo de retinopatía por hidroxicloroquina y cloroquina depende principalmente de la dosis acumulada y la duración del tratamiento.

Palabras clave: Hidroxicloroquina; Cloroquina; Retinopatía; COVID-19.

\section{Introdução}

O surgimento e dispersão do vírus SARS-CoV-2 pelo mundo e de sua infecção COVID-19, a partir, provavelmente, da Cidade de Wuhan na China desde dezembro de 2019, causou uma emergência de saúde pública de importância mundial ao se alastrar por todos os continentes. Essa doença até 24/10/2021 acarretou no mundo todo mais de 244 milhões de casos e 4,9 milhões de mortes, só no Brasil foram mais de 21,7 milhões de casos e 600 mil mortes (Worldometer, 2021).

Devido a essa emergência de saúde pública internacional, há uma intensa busca por meios de se conter a doença, o que inclui medidas individuais como o uso de máscaras em lugares públicos, distanciamento social, higiene constante das mãos (Singhal, 2020; Hu et al., 2020). Assim como medidas sociais como o rastreamento de contatos, testagem ativa, restrição de eventos e viagens, quarentena e lockdowns (Hussain et al., 2020)

Até o final de 2020, não se havia descoberto medicamentos ou vacinas eficazes contra a COVID-19 que fossem aprovadas por agências sanitárias para o uso da população em geral, embora inúmeras pesquisas clínicas em andamento apresentassem candidatos, e dada a gravidade da situação de saúde, pesquisadores, nesse meio tempo, buscaram em drogas disponíveis no mercado pelo potencial tratamento contra o novo coronavírus. Um dos tratamentos levantados e divulgados, à época, o qual em testes in vitro havia surtido efeito contra o SARS-CoV-2 foi o com HCQ e CQ

O Ministério da Saúde do Brasil (MS) em 2020, considerando esses estudos que demonstravam in vitro eficácia dos fármacos contra o novo coronavírus, adotou a CQ e HCQ no tratamento precoce contra a COVID-19 por meio do “Orientações do Ministério da Saúde para Manuseio Medicamentoso Precoce de Pacientes com Diagnóstico da COVID-19”. Nesse documento se orienta a prescrição de CQ ou HCQ por 14 dias com azitromicina para pacientes.

Contudo, sabe-se devido ao uso da HCQ/CQ na terapia de outras moléstias, como no lúpus eritematoso sistêmico, que essas medicações precipitam efeitos colaterais diversos, como no sistema cardíaco, o prolongamento do intervalo QT e arritmia ventricular, e outros de cunho oftalmológico, tal qual toxicidade retinal, retinopatia e até cegueira.

Portanto, diante da ampla utilização e da recomendação no Brasil do uso destes fármacos contra a COVID-19, a 
gravidade das alterações oculares que podem ser geradas e ampla população que se expôs, vimos a relevância em examinar por meio de uma revisão integrativa de literatura, se há a possibilidade de ocorrer tais efeitos colaterais oftalmológicos, pensando os fatores de risco conhecidos para essas complicações como a duração da terapia, a dose acumulada entre outros e tendo como base a prescrição recomendada da HCQ e CQ no tratamento precoce contra COVID-19 pelo MS.

\section{Metodologia}

Trata-se de uma revisão integrativa, realizada como descrito por Souza (2010), que efetuou um levantamento de artigos na literatura pela plataforma PUBMED no mês de outubro de 2020. Para a pesquisa empregamos os descritores: "Hydroxychloroquine" OR "Chloroquine" AND "Ophthalmopathy" OR "Retinopathy”. Os critérios de inclusão de artigos foram: artigos escritos em português ou inglês e publicados entre 2015 e 2020. Os critérios de exclusão de artigos são: artigos que não abordem aspectos epidemiológicos ou fatores de riscos de ao menos um dos efeitos adversos oculares da HCQ/CQ;. artigos com o propósito de validar exames para diagnóstico ou prognóstico; artigos não disponíveis gratuitamente e relatos de caso.

Procedimentos de coleta de dados: Foi realizada uma busca por artigos na plataforma PUBMED no mês de outubro de 2020. Primeiramente, realizou-se uma leitura de títulos, do quais foram selecionados trabalhos pertinentes para a subsequente leitura dos resumos, os artigos filtrados por essas etapas foram os adequados para o propósito desse estudo e lidos.

Análise de dados: Os artigos selecionados pelas etapas descritas foram integralmente analisados, suas informações condensadas em tabela e enfim, confrontamos os dados encontrados sobre os efeitos colaterais oftalmológicos pelo uso de CQ/HCQ e as orientações preconizadas para o tratamento de COVID-19.

\section{Resultados}

A partir da seleção, foram selecionados 14 artigos, sendo 5 estudos de retrospectivos 3 de revisão de literatura, 3 estudos de coorte, 2 estudos transversais e 1 estudo prospectivo. Informações relevantes sobre os artigos selecionados foram descritas na Tabela 1.

Tabela 1 - Resultados dos artigos.

\begin{tabular}{|l|l|l|l|}
\hline $\begin{array}{l}\text { Nome do artigo. Ano. } \\
\text { Autores }\end{array}$ & Tipo de estudo & Achado/Resultado do Estudo & Fatores de Risco \\
\hline $\begin{array}{l}\text { Hydroxychloroquine } \\
\text { retinopathy. 2017. Yusuf } \\
\text { et al. }\end{array}$ & $\begin{array}{l}\text { Revisão de } \\
\text { literatura }\end{array}$ & $\begin{array}{l}\text { Recomendação de uso do peso real na } \\
\text { dosagem de HCQ/CQ e de testes visuais de } \\
\text { campo mais amplo para triagem em } \\
\text { asiáticos. }\end{array}$ & $\begin{array}{l}\text { Uso de tamoxifeno, Uso diário de HCQ } \\
\text { acima de 5 mg/kg/dia ou de CQ acima } \\
\text { de 2,3 mg/kg/dia, Uso das drogas por } \\
\text { mais de 5 anos, disfunção renal e } \\
\text { doenças maculares. }\end{array}$ \\
\hline $\begin{array}{l}\text { Challenges and cares to } \\
\text { promote rational use of } \\
\text { chloroquine and } \\
\text { hydroxychloroquine in the } \\
\text { management of } \\
\text { coronavirus disease 2019 } \\
\text { (COVID-19) pandemic: a } \\
\text { timely review. 2020. } \\
\text { Pereira. }\end{array}$ & $\begin{array}{l}\text { Revisão de } \\
\text { literatura }\end{array}$ & $\begin{array}{l}\text { Não há evidência que o uso de HCQ/CQ } \\
\text { tenha efeito preventivo aos efeitos do } \\
\text { COVID-19 }\end{array}$ & $\begin{array}{l}\text { Tratamento crônico e dose diária de } \\
\text { cloroquina acima de 2,3 mg/kg dia de } \\
\text { peso real. }\end{array}$ \\
\hline $\begin{array}{l}\text { Chloroquine And } \\
\text { Hydroxychloroquine } \\
\text { Toxicity. 2021. } \\
\text { Stokkermans et al. }\end{array}$ & $\begin{array}{l}\text { Revisão de } \\
\text { Literatura }\end{array}$ & $\begin{array}{l}\text { Maculopatia pericentral sem a clássica } \\
\text { retinopatia parafoveal (olhos de touro) é } \\
\text { mais comum em asiáticos. } \\
\text { Uso de anglos maiores em testes visuais de } \\
\text { screening em asiáticos. }\end{array}$ & $\begin{array}{l}\text { Dose cumulativa, Uso das drogas por } \\
\text { mais de 5 anos, uso de mais de 5 } \\
\text { mg/Kg/Dia por peso real de HCQ, } \\
\text { doença renal pré-existente e uso } \\
\text { concomitante de tamoxifeno. }\end{array}$ \\
\hline
\end{tabular}




\begin{tabular}{|c|c|c|c|}
\hline $\begin{array}{l}\text { Somatotype, the risk of } \\
\text { hydroxychloroquine } \\
\text { retinopathy, and safe } \\
\text { daily dosing guidelines. } \\
\text { 2018. Browning e Lee. }\end{array}$ & $\begin{array}{l}\text { Estudo } \\
\text { retrospectivo. }\end{array}$ & $\begin{array}{l}\text { Mulheres baixas e astênicas tem maior risco } \\
\text { de retinopatia por HCQ. } \\
\text { Recomendação da dosagem ser a menor } \\
\text { entre peso ideal e peso real. }\end{array}$ & $\begin{array}{l}\text { Dose diária acima de } 400 \mathrm{mg} / \mathrm{dia} \text { de } \\
\text { HCQ, Dose cumulativa, duração da } \\
\text { terapia, insuficiência renal e hepática, } \\
\text { sexo feminino, índice de massa corporal } \\
\text { menor, doença macular pré-existente e } \\
\text { uso concomitante de tamoxifeno }\end{array}$ \\
\hline $\begin{array}{l}\text { Frequency and Clinical } \\
\text { Characteristics of } \\
\text { Hydroxychloroquine } \\
\text { Retinopathy in Korean } \\
\text { Patients with } \\
\text { Rheumatologic Diseases. } \\
\text { 2017. Eo et al. }\end{array}$ & $\begin{array}{l}\text { Estudo } \\
\text { retrospectivo }\end{array}$ & $\begin{array}{l}\text { O Estudo demostrou que a frequência de } \\
\text { toxicidade retinal de HCQ avaliada pelas } \\
\text { recomendações revisadas da AAO foi maior } \\
\text { do que em relatórios anteriores. } \\
\text { Toxicidade retinal pericentral ou mista é } \\
\text { comum em pacientes coreanos, enfatizando } \\
\text { a necessidade de rastreio de uma aérea mais } \\
\text { ampla da retinal central nesta etnia. }\end{array}$ & $\begin{array}{l}\text { Em pacientes coreanos com doenças } \\
\text { reumatológicas, dose cumulativa } \geq 600 \\
\mathrm{~g} \text { e a duração total da ingestão de HCQ } \\
\geq 72 \text { meses. Dose diária média de }>5,6 \\
\mathrm{mg} / \text { dia. }\end{array}$ \\
\hline $\begin{array}{l}\text { Incidence of and risk } \\
\text { factors for chloroquine } \\
\text { and hydroxychloroquine } \\
\text { retinopathy in Thai } \\
\text { rheumatologic patients. } \\
\text { 2016. Nuanpan et al. }\end{array}$ & $\begin{array}{l}\text { Estudo coorte } \\
\text { retrospectivo }\end{array}$ & $\begin{array}{l}\text { Os pacientes que tomam } \mathrm{CQ} / \mathrm{HCQ} \text { por um } \\
\text { longo tempo devem passar por rastreio } \\
\text { oftalmológico anual, após o exame de base. }\end{array}$ & $\begin{array}{l}\text { Foram citados como estatisticamente } \\
\text { relevantes }(P<0.05) \text { para retinopatia por } \\
\text { CQ: Idade, Peso real e dose } \\
\text { acumulativa. }\end{array}$ \\
\hline $\begin{array}{l}\text { Incidence of blindness in a } \\
\text { population of rheumatic } \\
\text { patients treated with } \\
\text { hydroxychloroquine. } \\
\text { 2019. Singh et al. }\end{array}$ & $\begin{array}{l}\text { Revisão } \\
\text { retrospectiva } \\
\text { longitudinal }\end{array}$ & $\begin{array}{l}\text { A toxicidade macular induzida por HCQ é } \\
\text { rara na prática clínica de rotina (taxa de } \\
\text { incidência pessoa-tempo de } 0,18 \text { por } 1000 \text { ), } \\
\text { foi identificada em três pacientes com }>18 \\
\text { anos de tratamento e não foi associada à } \\
\text { perda visual funcional. }\end{array}$ & $\begin{array}{l}\text { Diagnóstico reumatológico, uso } \\
\text { concomitante de tamoxifeno e duração } \\
\text { do uso de HCQ }\end{array}$ \\
\hline $\begin{array}{l}\text { Fluorescence Lifetimes in } \\
\text { Patients With } \\
\text { Hydroxychloroquine } \\
\text { Retinopathy. } 2019 . \\
\text { Solberg et al. }\end{array}$ & $\begin{array}{l}\text { Estudo } \\
\text { prospectivo de } \\
\text { caso controle }\end{array}$ & $\begin{array}{l}\text { O estudo demonstra que a retinopatia por } \\
\text { CHQ tem um padrão de fluorescência } \\
\text { característico no exame de fluorescência. }\end{array}$ & $\begin{array}{l}\text { Terapia com HCQ de longo prazo } \\
\text { (duração }>5 \text { anos de uso), doses mais } \\
\text { altas }(>5,0 \mathrm{mg} / \mathrm{kg} \text { ), doenças } \\
\text { reumatológicas e uso de tamoxifeno. }\end{array}$ \\
\hline $\begin{array}{l}\text { Current screening } \\
\text { practice in patients under } \\
\text { long-term } \\
\text { hydroxychloroquine } \\
\text { medication in Taiwan. } \\
\text { 2019. Chu-Yu et al. } \\
\end{array}$ & $\begin{array}{l}\text { Estudo coorte } \\
\text { retrospectivo }\end{array}$ & $\begin{array}{l}\text { É importante ao prescrever HCQ agendar } \\
\text { os exames de triagem oftalmológico tanto } \\
\text { inicial quanto anual e informar aos } \\
\text { pacientes sobre as possíveis complicações } \\
\text { oculares graves, mesmo em pacientes em } \\
\text { tratamento com menos de } 5 \text { anos }\end{array}$ & $\begin{array}{l}\text { A duração do uso de HCQ (por mais de } \\
5 \text { anos) e uma dose diária }>5 \mathrm{mg} / \mathrm{kg} / \mathrm{dia}\end{array}$ \\
\hline $\begin{array}{l}\text { Evaluation of toxic } \\
\text { retinopathy caused by } \\
\text { antimalarial medications } \\
\text { with spectral domain } \\
\text { optical coherence } \\
\text { tomography. 2019.Cabral } \\
\text { et al. }\end{array}$ & $\begin{array}{l}\text { Estudo } \\
\text { transversal }\end{array}$ & $\begin{array}{l}\text { O uso de tomografia de coerência óptica de } \\
\text { domínio espectral foi essencial para o } \\
\text { diagnóstico de retinopatia tóxica } \\
\text { antimalárica em estágio inicial }\end{array}$ & $\begin{array}{l}\text { Fatores maiores: Uso por mais de } 5 \\
\text { anos, nefropatia, uso concomitante de } \\
\text { tamoxifeno, maculopatia prévia, dose } \\
\text { de HCQ maior que } 5 \mathrm{mg} / \mathrm{Kg} \text { de peso } \\
\text { real ou de CQ maior que } 2,3 \mathrm{mmg} / \mathrm{Kg} \\
\text { de peso real } \\
\text { Fatores menores: Idade de mais de } 60 \\
\text { anos, presença de doença hepática e } \\
\text { fatores genéticos. }\end{array}$ \\
\hline $\begin{array}{l}\text { Chloroquine retinopathy: } \\
\text { pattern of presentation in } \\
\text { Ibadan, Sub-Sahara } \\
\text { Africa. } 2016 \text {. Oluleye et } \\
\text { al. }\end{array}$ & $\begin{array}{l}\text { Estudo } \\
\text { retrospectivo }\end{array}$ & $\begin{array}{l}\text { A maculopatia em olho de boi e extensa } \\
\text { degeneração retiniana e macular com } \\
\text { atrofia óptica são as principais } \\
\text { manifestações de retinopatia por CQ na } \\
\text { África Subsaariana. }\end{array}$ & $\begin{array}{l}\text { Dose acumulada entre } 100 \text { a } 300 \mathrm{~g} \text { causa } \\
\text { toxicidade }\end{array}$ \\
\hline $\begin{array}{l}\text { An audit of the use of } \\
\text { hydroxychloroquine in } \\
\text { rheumatology clinics. } \\
2018 \text {. Worth et al. }\end{array}$ & $\begin{array}{l}\text { Estudo } \\
\text { transversal }\end{array}$ & $\begin{array}{l}\text { A terapia com HCQ está sendo usada por> } \\
5 \text { anos em } 29 \% \text { dos pacientes com doenças } \\
\text { reumáticas, com doses maiores do que as } \\
\text { recomendadas em aproximadamente } 10 \% \\
\text { dos pacientes. }\end{array}$ & $\begin{array}{l}\text { Doses diária de HCQ acima de } 5 \\
\mathrm{mg} / \mathrm{kg} / \text { dia por peso real , duração da } \\
\text { terapia. }\end{array}$ \\
\hline $\begin{array}{l}\text { Prevalence of } \\
\text { hydroxychloroquine } \\
\text { retinopathy using } 2018 \\
\text { Royal College of } \\
\text { Ophthalmologists } \\
\end{array}$ & $\begin{array}{l}\text { Estudo } \\
\text { retrospectivo }\end{array}$ & $\begin{array}{l}\text { A prevalência da retinopatia por } \\
\text { hidroxicloroquina ( } 1,6 \%) \text { observda é menor } \\
\text { do que a prevalência relatada anteriormente } \\
\text { de } 7,5 \% \text {, conforme relatado por Melles e } \\
\text { Marmor, decorre dos critérios diagnósticos }\end{array}$ & A dose e a duração do tratamento \\
\hline
\end{tabular}




\begin{tabular}{|c|c|c|c|}
\hline $\begin{array}{l}\text { diagnostic criteria. } 2021 . \\
\text { Marshall et al. }\end{array}$ & & diferentes & \\
\hline $\begin{array}{l}\text { Two years' experience of } \\
\text { screening for } \\
\text { hydroxychloroquine } \\
\text { retinopathy. } 2021 . \\
\text { Gobbett et al. }\end{array}$ & Estudo de coorte & $\begin{array}{l}\text { O artigo relata menor prevalência de } \\
\text { retinopatia }(0,3 \%) \text { que o reportado pela } \\
\text { Academia Real de Oftalmologia ( } 7,5 \% \text { após } \\
5 \text { anos de uso) usando os seus mesmos } \\
\text { critérios. O qual atribuem a menor } \\
\text { quantidade de pacientes que tomam altas } \\
\text { doses de HCQ. }\end{array}$ & $\begin{array}{l}\text { Duração do uso de HQC por }>5 \text { anos, } \\
\text { diagnostico de doenças reumatológicas, } \\
\text { dose diária }>5 \mathrm{mg} / \mathrm{kg} / \text { dia por peso real e } \\
\text { uso concomitante de tamoxifeno. }\end{array}$ \\
\hline
\end{tabular}

Fonte: Autores.

\subsection{Duração da terapia}

Em 12 estudos, foi citado a duração do uso contínuo de $\mathrm{HCQ} / \mathrm{CQ}$ como fator de risco para retinopatias. Destes sete afirmavam que a administração por mais de 5 anos de HCQ/CQ configura como um risco, devido a prevalência de 7,5\% de retinopatias neste recorte, o qual aumenta para 20\% depois de 20 anos de terapia (Yusuf et al., 2017; Stokkermnas et al., 2021; Solberg et al., 2019; Chu-Yu et al., 2019; Cabral et al., 2019; Worth et al., 2018; Gobbett et al., 2020). Eo et al (2017) que realizou um estudo retrospectivo em pacientes coreanos afirma que a administração por mais de 3 anos de HCQ constitui em um fator de risco significante.

\subsection{Método de dosagem}

Em 5 estudos, foi citado o método de dosagem de HCQ/CQ como um fator de risco ou para estabelecer uma predileção entre os métodos. Três estudos afirmavam que a dosagem é melhor desempenhada por peso real em detrimento de por peso ideal, pois a dosagem por peso real é um preditor superior de toxicidade retiniana (Yusuf et al., 2017; Pereira, 2020; Stokkermans et al., 2021). Browning e Lee (2018) recomendam que a dosagem utilizada deva ser a menor entre a por peso real ou a por peso ideal, pois garante maior proteção tanto a pacientes astênicos e curtos quanto a mulheres, curtas e obesas.

\subsection{Dose diária ingerida}

Em 11 estudos, foi citado a dose tomada diariamente como um fator de risco. 9 estudos concluíram que doses diárias acima de 5,0 mg/kg de HCQ ou 2,3 mg/kg de CQ por peso real constituem um risco (Yusuf et al., 2017; Pereira, 2020; Stokkermnas et al., 2021. Eo et al., 2017; Nuanpan et al., 2016; Singh et al., 2019; Solberg et al., 2019; Worth et al., 2018; Gobbet et al., 2020). 1 estudo afirmou que o risco de retinopatia com HCQ é 2 a 3 vezes maiores com doses maiores que 5,0 comparado as de 4,0 mg/kg/dia por peso real (Yusuf et al., 2017) e Stokkermans et al (2021) alega que doses acima de 5,0 $\mathrm{mg} / \mathrm{kg} / \mathrm{dia}$ de HCQ aumenta o odds de retinopatia em 5,7 vezes.

\subsection{Tipo Físico}

Em 2 estudos, foi citado o tipo físico como um fator risco ou proteção. De acordo com o método de dosagem escolhido é conferido maior proteção aos riscos de retinopatia por HCQ/CQ a certo somatótipo. A dosagem por peso ideal confere segurança aos biotipos com baixa estatura e obesos e a dosagem por peso real aos de baixa estatura e magros de receberem doses elevadas (Stokkermans et al., 2021). Browning e Lee (2018) afirmam que as mulheres, astênicas e curtas são as com maior risco de retinopatia devido as diretrizes anteriores a 2016 recomendarem dosagem por peso ideal.

\subsection{Dose Cumulativa}

Em 8 estudos, foi referida a dose cumulativa como fator de risco para retinopatia por HCQ/CQ. Em 3 estudos encontraram correlação significativa entre a dose cumulativa de HCQ e a retinopatia (Chu-Yu et al., 2019; Cabral et al., 2019; 
Browning \& Lee, 2018). A dose cumulativa maior ou igual $600 \mathrm{~g}$ foi um fator de risco significativo para retinopatia HCQ. (EO et al, 2017). Conforme Stokkermans et al. (2021) revelou o indicador mais importante de toxicidade era a duração do uso (dose cumulativa). Atinge-se uma dose cumulativa de 1000 gramas (1 quilograma) em 7 anos, quando se toma a dose mais comumente prescrita de $400 \mathrm{mg}$ por dia. Foi observado também que houve uma relação estatisticamente significativa com uma dose cumulativa inferior a $460 \mathrm{~g}$ em pacientes com retinopatia CQ. O estudo não encontrou nenhuma relação estatisticamente significativa com a dose cumulativa na incidência de retinopatia HCQ (Nuanpan et al., 2016). De acordo, as diretrizes da AAO de 2011 declararam que uma dose cumulativa de mais de $1000 \mathrm{~g}$ aumenta o risco de retinopatia. No entanto, as conclusões tiradas sobre a importância da dose cumulativa são inconsistentes, sendo omitido como um fator de risco importante nas diretrizes da AAO de 2016 recentes (Yusuf et al., 2017).

\subsection{Raça}

Dos artigos selecionados para o estudo foi citado a raça como um fator de risco para toxicidade retinal por uso de HQC e CQ diferenciando o acometimento de acordo os dados demográficos. Um dos estudos afirma que asiáticos têm retinopatia de campo mais amplo, diferente das demais populações (Yusuf et al., 2017). Segundo Eo et al. (2017), a maioria dos estudos relatou toxicidade retinal de HCQ ocorrida na região parafoveal. No entanto, eles mostraram um novo padrão de dano retiniano na região pericentral, e esse dano retiniano pericentral foi mais comum em pacientes asiáticos. Outro estudo especifica que o CQ se liga ao pigmento melanina do EPR, o que, talvez, leve à toxicidade e danos ao EPR. Como as populações tailandesas têm mais pigmento melanina do que os caucasianos, o CQ pode se acumular mais no EPR dos tailandeses que nos olhos caucasianos (Nuanpan et al., 2016).

\subsection{Sexo}

Apenas 2 artigos mostram relevância do gênero para o risco de retinopatia HCQ/CQ. Oluleye et al. (2016) alegou que a automedicação com cloroquina foi a principal causa de retinopatia nos pacientes da África subsaariana, os homens foram os mais afetados nesse estudo por serem os maiores usuários de CQ. Em outro estudo observou-se que mulheres astênicas e baixas corriam maior risco. As mulheres eram significativamente mais propensas a ter retinopatia por HCQ do que os homens (Browning \& Lee, 2018).

\subsection{Uso de Tamoxifeno}

Dos 14 artigos selecionados para o estudo, 9 citaram o uso de Tamoxifeno como fator de risco para toxicidade retiniana por HCQ. Yusuf et al. (2017) afirma que o tamoxifeno foi investigado como potencialmente causador de uma maculopatia dependente da dose que pode, ao mesmo tempo, aumentar o risco de toxicidade do HCQ. A terapia com tamoxifeno aumenta o risco de toxicidade retinal, os antiácidos e a argila de caulim reduzem a atividade de CQ / HCQ e o paciente deve tomar os medicamentos com 4 horas de intervalo (Stokkermans et al., 2021). Quatro estudos apontam que o uso concomitante de Tamoxifeno confere um risco maior de toxicidade retinal do HCQ (Nuanpan et al., 2016; Singh et al., 2019; Solberg et al., 2019; Worth et al., 2018). Dois artigos citaram o Tamoxifeno como fator de risco, mas não mostraram risco estabelecido e especificado no artigo quando usado com a HCQ (Marshall et al., 2021; Gobbet et al., 2020).

\subsection{Comorbidades}

Dos 14 artigos selecionados para este estudo, 10 abordam que pacientes com doenças reumatológicas em uso contínuo de HCQ/CQ tem mais chances de desenvolver doenças relacionadas a retina. Dois estudos relatam que doenças autoimunes, dentre estas Lúpus, Síndrome de Sjogren, Artrite Reumatoide e outras doenças do tecido conjuntivo que fazem o tratamento 
apenas com HCQ tem risco menores de desenvolver retinopatias e alertam que a retirada do HCQ está associada a um risco aumentado de desenvolver uma exacerbação da doença em pacientes estáveis. (Browning \& Lee, 2018; Tangtavorn et al., 2016). Quatro estudos expõem que pacientes com doenças reumatológicas que fazem uso prolongado de CQ e HCQ devem fazer a detecção precoce da retinopatia, pois o dano pode progredir mesmo após a interrupção do medicamento devido à lenta eliminação do medicamento do corpo. Assim, a triagem para toxicidade retinal precoce é crucial para detectar sinais de maculopatia em uma "fase pré-clínica" para prevenir perda visual severa por meio da descontinuação precoce da medicação (Solberg et al., 2019; Cabral et al., 2019; Eo et al., 2017 e Worth et al., 2018). Um estudo relata que a maioria dos pacientes com doenças autoimunes que fazem uso a longo prazo de CQ e HCQ desenvolvem retinopatia antes de 5 anos e sugere que os pacientes devem ser submetidos a triagem oftalmológica anualmente (Tangtavorn et al., 2016). Um estudo que trata do índice de cegueira na população de pacientes reumáticos tratados com hidroxicloroquina relata que a toxicidade macular induzida por HCQ é rara, e que para que esta aconteça é necessário terapia prolongada (mais de 18 anos) e que mesmo assim não está necessariamente associada à perda visual funcional. Seus achados sugerem que as condições comórbidas comuns na AR e no LES contribuem substancialmente para a perda de visão e não devem ser ignoradas (Singh et al., 2019).

\section{Discussão}

Durante a pandemia de COVID-19, a HCQ e a CQ ganharam relevância devido as recomendações de seu uso no tratamento contra o coronavírus. O MS preconizou o seu uso no Brasil e vários estudos respaldaram a decisão ministerial à época, embora a utilização para este fim seja controversa. Dado a importância e uso destes medicamentos durante a pandemia em largas populações, fez-se necessário analisar se os efeitos colaterais oftalmológicos destes fármacos poderiam afetar os pacientes que realizaram o tratamento preconizado à época.

No tocante a duração da terapia medicamentosa com HCQ/CQ, houve concordância entre a maioria dos autores de que o uso contínuo por mais de 5 anos constitui um fator de risco, e que a prevalência de retinopatia nessa população é de 7,5\%, estes estudos se embasavam nas recomendações da AAO de 2016 quanto o rastreamento de retinopatia por CQ e HCQ, o qual se apoia em uma análise epidemiológica em 2361 pacientes. Eo et al. (2017) diverge dos demais autores, em um estudo retrospectivo com 310 pacientes coreanos com doenças reumatológicas, ao afirmar que a administração por mais de 3 anos de HCQ constitui um fator de risco significante e demonstra uma taxa de prevalência de retinopatia após 5 anos de uso de 5,2\%. Tal discordância de prevalência é observada em outros estudos como o de Chu-Yu et al. (2019), que realizou um estudo coorte em toda população taiwanesa, em que relata uma prevalência de 3,9\%. Essas divergências em prevalência se devem tanto a diferente etnia das populações analisadas quanto aos critérios diagnósticos distintos utilizados em cada pesquisa.

Quanto ao método de dosagem a maior parte dos autores concordam, a partir de 2016, em orientar a prescrição por peso ideal, isso se deve a recomendação da AAO em 2016, na qual afirma que esse método é superior como preditor de toxicidade retiniana. Contudo, Browning e Lee (2018) em seu estudo retrospectivo com 565 pacientes discorda dessa afirmação, ao não observar essa correlação em sua análise, além de recomendar que a dosagem seja realizada com a menor dose entre a por peso real e peso ideal no intuito de prevenir a sobredosagem.

No que diz respeito a dose cumulativa, como fator de risco para retinopatia, essa revisão encontrou que há concordância entre a maioria dos autores. Entretanto, divergem quanto a dose acumulada necessária para se ter um risco significativo de retinopatia por HCQ/CQ. É evidente, também, que a dose cumulativa precipitadora de retinopatia pelo uso de CQ é inferior ao da HCQ conforme Stokkermans et al. (2021). Por outro lado, outros autores não encontraram nenhuma relação estatisticamente significativa com a dose cumulativa na incidência de retinopatia HCQ. (Nuanpan et al., 2016; Yusuf et al., 2017).

A respeito da dose diária ingerida há um consenso entre os autores de que a dose diária acima de 5,0 $\mathrm{mg} / \mathrm{kg}$ de HCQ 
ou 2,3 $\mathrm{mg} / \mathrm{kg}$ de CQ por peso real constituem um fator de risco. Yusuf et al. (2017) acrescenta que o risco de desenvolvimento de retinopatia por HCQ é 2 a 3 vezes maiores com doses maiores a 5,0 mg/kg/dia comparado as de 4,0 mg/kg/dia por peso real e Stokkermans et al (2021) alega que doses acima de 5,0 mg/kg/dia por peso real aumento o odds de retinopatia em 5,7 vezes.

No que tange o tipo físico, a proteção conferida a determinado somatótipo é garantida pela escolha do método de dosagem. A dosagem por peso real proporciona segurança a pessoas curta e obesas quanto o risco de sobredosagem, e a por peso ideal assegura o mesmo aos pacientes magros e baixos. Browning e Lee (2018) orienta por isso que seja dada a menor dose entre os dois métodos.

Em relação a raça, os estudos relataram que a toxicidade retiniana de HCQ ocorre na região parafoveal, exceto em pacientes asiáticos em que o dano retiniano na região pericentral foi mais comum (Eo et al., 2017). Outro estudo especifica que o CQ pode se acumular mais no EPR dos tailandeses do que nos caucasianos, segundo Nuapan et al. (2016).

Sobre o gênero, essa revisão mostra pouca relevância deste fator para o risco de retinopatia por HCQ/CQ. De acordo com Oluleye et al. (2016) relatam os homens foram os mais afetados por retinopatia por serem os maiores usuários da automedicação com CQ na África subsaariana. Todavia, Browning e Lee (2018) alegam que as mulheres eram significativamente mais propensas a ter retinopatia por HCQ do que os homens apesar das causas não estarem claras.

O uso concomitante do Tamoxifeno, na maioria dos artigos estudados, foi relatado como potencialmente causador de uma maculopatia dependente da dose que pode, ao mesmo tempo, aumentar o risco de toxicidade do HCQ. A minoria dos estudos citava o Tamoxifeno como fator de risco, porém não especificam o risco quando usado com a HCQ.

Referente as comorbidades, os autores concordam que pacientes com doenças reumatológicas em uso contínuo de HCQ tem mais chances de desenvolver doenças relacionadas a retina. Ainda afirmam que os pacientes com doenças reumatológicas que fazem uso prolongado de CQ e HCQ devem fazer a deteç̧ão precoce da retinopatia, pois o dano pode progredir mesmo após a interrupção do medicamento (Solberg et al., 2019; Cabral et al., 2019; Eo et al., 2017 e Worth et al., 2018).

As principais variáveis relacionadas à prescrição para tratamento precoce de COVID-19 são a dose acumulativa e a duração do tratamento. O tratamento preconizado pelo MS durante 2020 (Brasil, 2020) incluía HCQ/CQ em um regime de 14 dias, no qual totalizava 7,6 g de HCQ ou 9,5 g de CQ. Em nossa revisão, constatamos que a retinopatia medicamentosa é observada a partir da uma dose acumulativa de ao menos 600g de HCQ (Eo et al., 2017) ou de ao menos 460g de CQ (Stokkermanas et al., 2021), em uma terapia com duração de ao menos 3 anos (Eo et al., 2017). Como o tratamento preconizado pela MS tinha valores inferiores a esses dois parâmetros, não se espera que os pacientes desenvolvam alterações oftalmológicas (toxicidade ou retinopatia) decorrentes do uso de HCQ/CQ por esse protocolo.

\section{Considerações Finais}

Na busca por terapias medicamentosas contra o COVID-19 durante a epidemia, alguns autores e entidades de saúde e sanitárias viram na HCQ/CQ uma opção de tratamento, contudo há riscos associados a ingestão desses medicamentos, como a toxicidade retinal, retinopatia e, até a cegueira. Na presente revisão, constatamos que os principais fatores de risco para o desenvolvimento de retinopatia por $\mathrm{HCQ} / \mathrm{CQ}$ são um consenso para os autores, e são eles a duração da terapia, a dose acumulativa e a dose diária ingerida. A idade não foi um citado como fator de risco nos estudos abrangidos.

Os dados levantados nessa pesquisa sugerem que o tratamento precoce orientado contra o coronavírus pelo MS com HCQ/CQ não induza a toxicidade retinal ou retinopatia, pois o tempo de terapia e, principalmente, a dose acumulativa dos fármacos são inferiores aos níveis considerados prejudiciais.

Esse estudo é importante devido à grande população que foi submetida a esse tratamento e a relevância em saber se esses indivíduos poderiam estar com risco de sofrer com efeitos colaterais oculares do HCQ/CQ. A presente revisão é limitada 
pela pequena quantidade de estudos que aborda e busca esclarecer sobre um tema de saúde atual e emergente.

Averiguamos que há lacunas há serem abordadas em futuros estudos sobre o tema, como a multiplicidade de definições utilizadas para o diagnóstico de toxicidade retinal, o reduzido tamanho das amostras populacionais dos estudos, e a falta de estudo originais multicêntricos que analisem grandes amostras de populações de etnias distintas, dado que há evidências que apontam diferenças no desenvolvimento destes efeitos colaterais de acordo com a origem étnica do paciente, assim como ajudaria a definir acuradamente a real taxa de prevalência da toxicidade retinal entre utilizadores de HCQ/HCQ.

\section{Referências}

Browning, D. J., \& Lee, C. (2018). Somatotype, the risk of hydroxychloroquine retinopathy, and safe daily dosing guidelines. Clinical Ophthalmology (Auckland, NZ), 12, 811. https://doi.org/10.2147/OPTH.S163451

Cabral, R. T. D. S., Klumb, E. M., Couto, M. I. N. N., \& Carneiro, S. (2018). Evaluation of toxic retinopathy caused by antimalarial medications with spectral domain optical coherence tomography. Arquivos brasileiros de oftalmologia, 82, 12-17. https://doi.org/10.5935/0004-2749.20190002

Eo, D. R., Lee, M. G., Ham, D. I., Kang, S. W., Lee, J., Cha, H. S., Koh, E. \& Kim, S. J. (2017). Frequency and clinical characteristics of hydroxychloroquine retinopathy in Korean patients with rheumatologic diseases. Journal of Korean medical science, 32(3), 522-527. https://doi.org/10.3346/jkms.2017.32.3.522

Gobbett, A., Kotagiri, A., Bracewell, C., \& Smith, J. (2021). Two years' experience of screening for hydroxychloroquine retinopathy. Eye, 35(4), 1171-1177. https://doi.org/10.1038/s41433-020-1028-4

Hu, B., Guo, H., Zhou, P., \& Shi, Z. L. (2021). Characteristics of SARS-CoV-2 and COVID-19. Nature Reviews Microbiology, 19(3), 141-154. https://doi.org/10.1038/s41579-020-00459-7

Hussain, A., Yadav, S., Hadda, V., Suri, T. M., Tiwari, P., Mittal, S., Madan, K. \& Mohan, A. (2020). Covid-19: a comprehensive review of a formidable foe and the road ahead. Expert review of respiratory medicine, 14(9), 869-879. https://doi.org/10.1080/17476348.2020.1782198

Marmor, M. F. (2020). COVID-19 and chloroquine/hydroxychloroquine: is there ophthalmological concern?. American journal of ophthalmology, 216, A1A2. https://doi.org/10.1016/j.ajo.2020.03.028.

Marmor, M. F., Kellner, U., Lai, T. Y., Melles, R. B., \& Mieler, W. F. (2016). Recommendations on screening for chloroquine and hydroxychloroquine retinopathy (2016 revision). Ophthalmology, 123(6), 1386-1394. https://doi.org/10.1016/j.ophtha.2016.01.058.

Marshall, E., Robertson, M., Kam, S., Penwarden, A., Riga, P., \& Davies, N. (2021). Prevalence of hydroxychloroquine retinopathy using 2018 Royal College of Ophthalmologists diagnostic criteria. Eye, 35(1), 343-348. https://dx.doi.org/10.1038\%2Fs41433-020-1038-2

Ministério da Saúde do Brasil. (2020). Orientações do Ministério da Saúde para Manuseio Medicamentoso Precoce de Pacientes com Diagnóstico da COVID19. http://www.mpgo.mp.br/portal/arquivos/2020/06/16/08_50_12_691_MS_manuseio_de_medicamentos_COVID_FINAL_15JUNHO.pdf

Oluleye, T. S., Babalola, Y., \& Ijaduola, M. (2016). Chloroquine retinopathy: pattern of presentation in Ibadan, Sub-Sahara Africa. Eye, 30(1), 64-67. https://doi.org/10.1038/eye.2015.185

Pereira, B. B. (2020). Challenges and cares to promote rational use of chloroquine and hydroxychloroquine in the management of coronavirus disease 2019 (COVID-19) pandemic: a timely review. Journal of Toxicology and Environmental Health, Part B,23(4), 177-181. https://doi.org/10.1080/10937404.2020.1752340

Singhal, T. (2020). A review of coronavirus disease-2019 (COVID-19). The indian journal of pediatrics, 87(4), 281-286. https://doi.org/10.1007/s12098-02003263-6

Solberg, Y., Dysli, C., Möller, B., Wolf, S., \& Zinkernagel, M. S. (2019). Fluorescence lifetimes in patients with hydroxychloroquine retinopathy. Investigative ophthalmology \& visual science, 60(6), 2165-2172. https://doi.org/10.1167/iovs.18-26079

Souza, M. T. D., Silva, M. D. D., \& Carvalho, R. D. (2010). Revisão integrativa: o que é e como fazer. Einstein (São Paulo), 8, 102-106. https://doi.org/10.1590/S1679-45082010RW1134

Stokkermans, T. J., Goyal, A., Bansal, P., \& Trichonas, G. (2021). Chloroquine And Hydroxychloroquine Toxicity. In StatPearls. StatPearls Publishing. https://www.ncbi.nlm.nih.gov/books/NBK537086/

Tangtavorn, N., Yospaiboon, Y., Ratanapakorn, T., Sinawat, S., Sanguansak, T., Bhoomibunchoo, C., \& Laovirojjanakul, W. (2016). Incidence of and risk factors for chloroquine and hydroxychloroquine retinopathy in Thai rheumatologic patients. Clinical Ophthalmology (Auckland, NZ), 10, 2179. https://dx.doi.org/10.2147\%2FOPTH.S119872

Worldometer (2021). Covid-19 coronavirus pandemic. https://www.worldometers.info/coronavirus/

Worth, C., Yusuf, I. H., Turner, B., Gourier, H., Brooks, E. E., Mort, D. O., ... \& Luqmani, R. A. (2018). An audit of the use of hydroxychloroquine in rheumatology clinics. Rheumatology advances in practice, 2(1), rky013. https://doi.org/10.1093/rap/rky013

Yen, C. Y., Lee, P. H., Yen, J. C., Chen, C. C., Hu, H. Y., \& Tseng, P. C. (2019). Current screening practice in patients under long-term hydroxychloroquine medication in Taiwan: A nationwide population-based cohort study. Medicine, 98(14). https://dx.doi.org/10.1097\%2FMD.0000000000015122

Yusuf, I. H., Sharma, S., Luqmani, R., \& Downes, S. M. (2017). Hydroxychloroquine retinopathy. Eye, 31(6), 828-845. https://dx.doi.org/10.1038\%2Feye.2016.298 